\title{
INCORPORACIÓN DE SISTEMAS MULTI-AGENTES EN EL APRENDIZAJE DE LA CULTURA E HISTORIA DE SANTA MARTA, COLOMBIA
}

\author{
INCORPORATION OF MULTI-AGENTS SYSTEMS IN LEARNING CULTURE AND \\ HISTORY OF SANTA MARTA, COLOMBIA
}

\author{
A. Pérez ${ }^{1}$, J. Taborda² \\ Recibido para publicación: 25 de mayo de 2014 - Aceptado para publicación: 7 julio de 2014
}

\begin{abstract}
RESUMEN
Este artículo plantea una forma de aprendizaje de la cultura e historia de Santa Marta, haciendo uso de técnicas de Inteligencia Artificial para crear un STI (Sistema Tutorial Inteligente) en el tema, que pueda adaptarse a los diferentes perfiles de usuarios y estilos de aprendizaje. Se muestra una aproximación metodológica y la estructura organizacional planteada para el modelo del usuario, teniendo en cuenta que ésta es la primera forma de adaptación del sistema. Los Sistemas Multi-Agentes es la técnica de Inteligencia Artificial que brinda la posibilidad de adaptación a los perfiles de usuario (estudiantes y turistas) además de los estilos de aprendizaje. Se pretende otorgar gran importancia al rol del turista el cual es muy importante en la ciudad de Santa Marta (distrito turístico, cultural e histórico), brindándole la posibilidad de aprender sobre la ciudad y ejercer turismo cultura en la misma, generando así una interacción dinámica entre el sistema y los usuarios (estudiantes y turistas), facilitando el proceso de aprendizaje por parte de los alumnos, así como la exploración cultural por parte de los turistas en la ciudad de Santa Marta.
\end{abstract}

Palabras clave: Sistema Tutorial Inteligente (STI), Inteligencia Artificial, Sistemas Multi-Agentes (SMA), Adaptación, Objetos de Aprendizaje, TAl

\begin{abstract}
This article proposes a way of learning about the culture and history of Santa Marta, using techniques to create an ITS (Intelligent Tutorial System) on the subject, which can adapt to different user profiles and learning styles. A methodological approach and organizational structure proposed for the user model is shown, considering that this is the first form of adaptation of the system. The Multi-Agent Systems is an Artificial Intelligence technique that offers the possibility to adapt to user profiles (students and tourists) as well as learning styles. It is intended to attach great importance to the role of tourist which is very important in the city of Santa Marta (tourism, cultural and historical district), providing the opportunity to learn about the city and exert tourism culture in itself, generating a dynamic interaction between the system and the users (students and tourists), facilitating the process of learning by students, as well as cultural exploration by tourists in the city of Santa Marta.
\end{abstract}

Keywords: Intelligent Tutorial System (ITS), Artificial Intelligence, Multi-Agent Systems (MAS), Adaptation, Learning Objects, TAl

1anikp.alvis@gmail.com, Universidad del Magdalena, Facultad de Ingeniería, Grupo MAGMA Ingeniería 2jtaborda@unimagdalena.edu.co, Universidad del Magdalena, Facultad de Ingeniería, Grupo MAGMA Ingeniería 


\section{INTRODUCCIÓN}

La enseñanza asistida por ordenador entre los años 70 y 90 fue altamente influenciada con la incorporación de técnicas de la Inteligencia Artificial en el desarrollo de sistemas y nuevas estrategiasdeenseñanza.Graciasalacercamiento de las Tecnologías de la Información (TICs) con los procesos de enseñanza-aprendizaje surgen los Cursos Virtuales Adaptativos (CVA) o Sistemas Tutoriales Inteligentes (STIs), cuyo objetivo básico es lograr el aprendizaje de un dominio específico del conocimiento por parte del estudiante mediante la utilización de herramientas propias de la informática y de la inteligencia artificial [1].

Salcedo apunta que los STIs generalmente persiguen objetivos de aprendizaje bien definidos y comúnmente aceptados, como conocimiento factual y habilidades procedurales - reflejo de una perspectiva objetivista del conocimiento- y que pueden medirse mediante tests estandarizados. Los desarrolladores de estos sistemas han tratado de demostrar, utilizando métodos tradicionales de aprendizaje, enseñanza y evaluación, que mejoran significativamente la velocidad y calidad del aprendizaje de los alumnos y hasta cierto punto han obtenido éxito en sus pretensiones [2].

El trabajo de investigación que se ha estado desarrollando y bajo el cual está enmarcado lo presentado en este artículo tiene como objetivo ofrecer una alternativa para promover una mejor experiencia de aprendizaje cultural e histórico de Santa Marta haciendo uso de técnicas de inteligencia artificial. La plataforma implementa Sistemas Multi-Agentes para identificar el tipo de usuario y el perfil de cada uno, detectando que objetos de aprendizaje son los más apropiados para presentar. Cada estudiante aprenderá según su perfil y capacidades, además el rol del turista se verá beneficiado ya que a través de este sistema podrá aprender aspectos importantes de la ciudad, dando a conocer el turismo cultural, sin necesidad de acudir a terceros como lo son guías turísticos y demás.
En el artículo presenta en la sección II algunos trabajos relacionados o que han servido como base para llevar a cabo el proyecto; en la sección III se describe la aproximación metodológica así como el modelo de usuario propuesto para que el sistema tenga en cuenta la existencia del perfil del turista además del estudiante, es decir, que sea adaptable dependiendo el tipo de usuario además del estilo de aprendizaje de los estudiantes, por último en la sección IV se presentan alunas conclusiones y trabajos futuros esperados.

\section{ESTADO DEL ARTE}

En cuanto al tema de la enseñanza cultural Pueyo [3] emplea una plataforma virtual que propone un acercamiento a la cultura española desde una perspectiva multidisciplinar.

España Multicultural es una invitación para que el alumno despierte la imaginación y emprenda un viaje virtual por la geografía, la lengua y la cultura española. Tras una introducción general, se plantea un recorrido por tres ciudades: Madrid, Barcelona y Bilbao. Los personajes más representativos, las imágenes, las palabras y los sonidos son guía en cada ciudad.

Para la creación de Sistemas tutoriales Inteligentes Cataldi et al. [4] proponen una arquitectura para los STIs que integran los aspectos más significativos de la tecnología de agentes lo cual sirve de ayuda para la creación del STI para la enseñanza-aprendizaje de la cultura e historia de Santa Marta. La arquitectura planteada considera la incorporación de agentes en los módulos del autor y del estudiante a fin de poder integrarlos al modelo considerando la base teórica de Perkins, los estilos de aprendizaje y las inteligencias múltiples de Gardner. Esta propuesta hace especial énfasis en el modelo del estudiante, el cual es muy completo y en la implementación de diferentes agentes tutores (interfaz, analizador de perfil, lenguaje natural, evaluado, etc) para llevar a cabo el proceso de enseñanza/aprendizaje de forma adaptativa. 
En otro trabajo Cataldi et al. [5] presenta un proyecto de investigación que lleva a integrar a los agentes inteligentes en el marco de desarrollo de los Sistemas Tutores Inteligentes (STI) a fin de obtener una arquitectura que soporte los marcos teóricos seleccionados: la teoría de agentes, las teorías de aprendizaje (estilos) y las estrategias de enseñanza a fin de articular la solución. Con este trabajo busca un sistema de alta modularidad que permita la reutilización de bloques principales. Para ello, se deben redefinir los módulos básicos a implementar con sus funcionalidades. De este modo se obtiene una arquitectura basada en agentes donde los módulos del tutor (MultiAgentes en este caso) y del estudiante se basa en los estilos de aprendizaje y en la teoría de las inteligencias múltiples de Gardner.

Arias et al. [6] Presenta una aproximación metodológica para el análisis, diseño y desarrollo de STIs bajo el paradigma de SMA en un entorno Web, que permite a los estudiantes realizar procesos de enseñanza/aprendizaje de forma personalizada y activa, a partir de este trabajo se ha basado la aproximación metodológica y el diseño del STI para la enseñanza-aprendizaje de la cultura e historia de Santa Marta. La validación de este modelo se realiza a través de la construcción de un prototipo computacional, llamado CIA. Este prototipo planifica inicialmente el aprendizaje de un estudiante específico para un curso virtual en el área de IA, adapta las evaluaciones y replanifica dinámicamente las actividades según los logros alcanzados o perdidos por un estudiante.

\section{APROXIMACIÓN METODOLÓGICA}

Los STI poseen una estructura básica, compuesta por 3 modelos específicos: Modelo de Dominio, Modelo de Estudiantes, Modelo Pedagógico. Teniendo en cuenta estos 3 modelos Arias Sánchez et al. [6] propuso una metodología basada en el paradigma de los Sistemas Multi-Agentes, la aproximación metodológica planteada para analizar y diseñar la plataforma de aprendizaje adaptativa sobre turismo cultural e histórico en la ciudad de Santa Marta es la siguiente.

En la etapa de análisis se contemplan los siguientes pasos

1) Especificación de la estructura organizacional

2) Definición de componentes principales del Modelo del Dominio

3) Definición de componentes principales del Modelo del Usuario

4) Definición de componentes principales del Modelo Pedagógico.

5) Selección de estrategias de planificación

6) En la etapa de diseño se contemplan los siguientes pasos:

7) Identificación de los actores que participan en el sistema

8) Diseño de un modelo Multi-Agente pedagógico adaptativo.

\section{DEFINICIÓN DE LA POBLACIÓN}

Una parte importante antes de establecer cualquier estructura del sistema es definir a quien se quiere enseñar. Esta es la pregunta más importante que ayuda a definir y delimitar la población a la que va dirigido el STI.

En Santa Marta, algunos colegios con formación técnica tienen entre sus programas de aprendizaje una especialidad enfatizada al turismo en la ciudad, abordando temas orientados a la venta de productos como son los planes turísticos en hoteles y agencias de viajes y guías turísticos en museos o lugares representativos histórica y culturalmente, como la Quinta de San Pedro Alejandrino.

Se ha escogido como población principal jóvenes entre 14-17 que cursan la especialidad en turismo en sus colegios y a los cuales se les quiere brindar una plataforma de aprendizaje colaborativo que les ayude a afianzar y extender sus conocimientos en el área de turismo, lo cual les ayudará directamente con lo cursado en sus aulas de clase. 
En cuanto a la población turista, ya que es un poco dispersa, se han planteado encuestas a través de redes sociales, las cuales ayudaran a definir sus necesidades y establecer qué tipo de información es la más solicitada por ellos, ya sea relacionada con lugares, datos culturales o guías turísticos en ciertas áreas de la ciudad, sabiendo que todos los temas estarán orientados a la cultura e historia de Santa Marta.

\section{ESTRUCTURA ORGANIZACIONAL PLANTEADA}

Con base en el modelo metodológico planteado por Arias Sánchez, se diseñó una estructura organizacional preliminar, en el caso de este proyecto no solo se trabaja con el usuario estudiantes sino también con el usuario turista.

La estructura organizacional propuesta tiene definido 4 modelos: Modelo de Dominio, Modelo de Estudiante, Modelo de Turista y Modelo Pedagógico como se muestra en la figura 1.
Laestructura u organización del modelo del dominio se da de tal manera que el conocimiento se divide por Cursos, los cuales se componen a su vez de Unidades Básicas de Aprendizaje (UBAs) que se encuentran conformadas por temas que tendrán guías asociadas, dichos temas tendrán asociados uno o varios Objetivos instruccionales a los cuales se podrán alcanzar por medio del desarrollo de una o varias actividades las cuales tienen uno o varios Objetos de Aprendizaje. En cuanto a las guías, se tiene una restricción dependiendo el tipo de usuario, para los usuarios turistas, inicialmente no tendrán que ver los temas relacionados con las unidades ya que solo podrán acceder a las guías asociadas con cada tema, esto se debe a que el turista no requiere un conocimiento tan profundo de los temas asociados con las unidades y tienen intereses específicos orientados más al turismo cultura que al aprendizaje de un curso completo sobre cultura e historia de Santa Marta. El usuario estudiante tendrá acceso a los cursos, temas, guías y todo lo demás disponibles para el desarrollo de su aprendizaje.

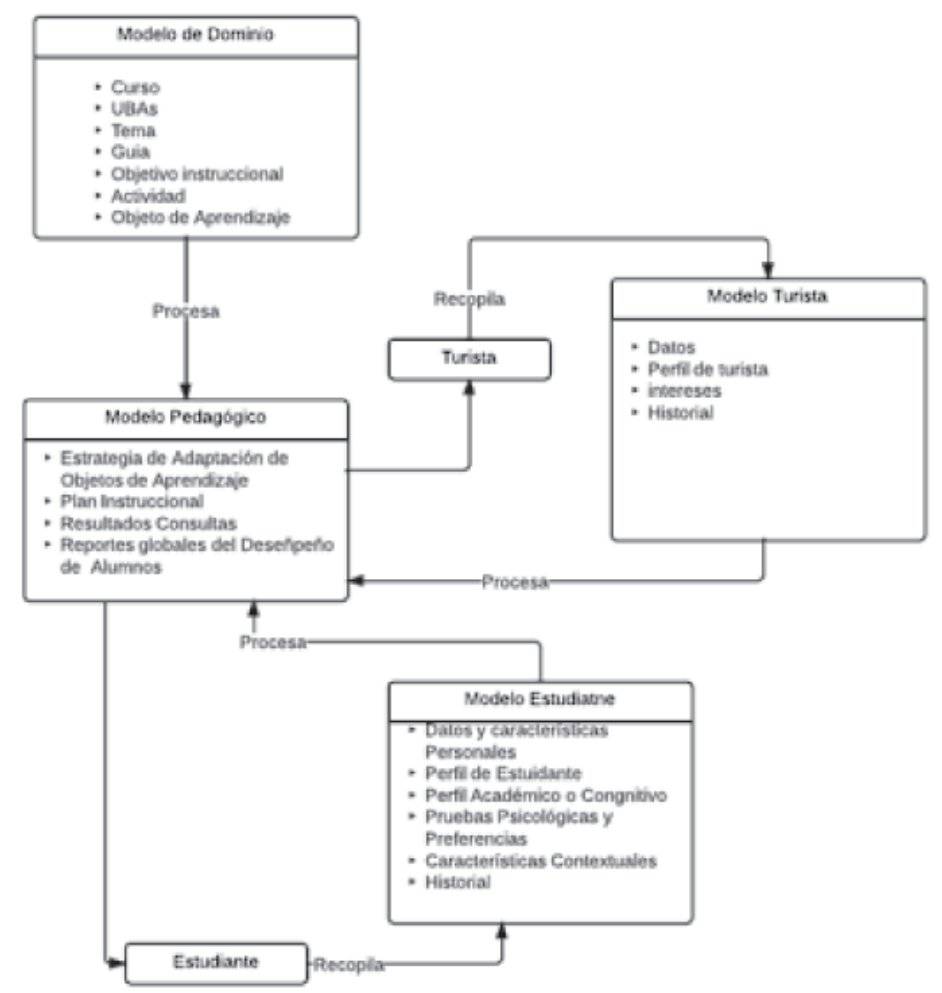

Figura 1. Estructura Organizacional 


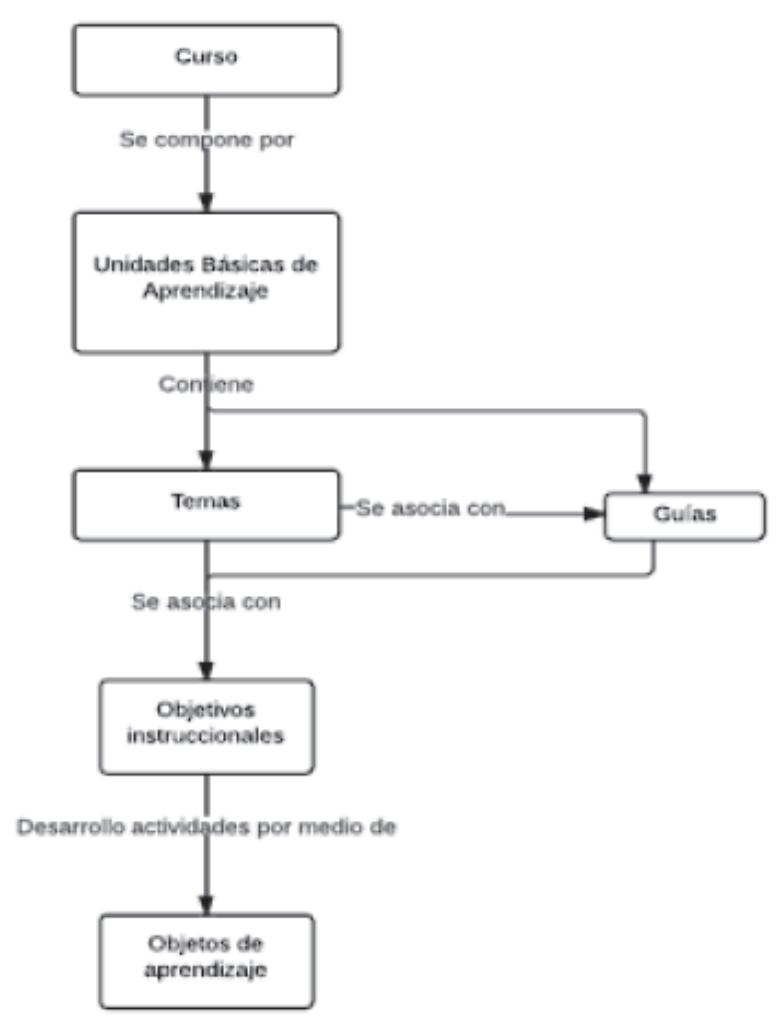

Figura 2. Módulo de Dominio

Por esta razón definir correctamente el modelo del estudiante y el modelo de turista es una etapa muy importante, estos deben ser explícitos y deben representar el conocimiento, las metas, los intereses y otras características que permitan al sistema distinguir entre varios usuarios como lo apunta Jiménez en [7].

Las características que se almacenan del usuario son relevantes en el desempeño del sistema así como sus interrelaciones. Se plantea inicialmente que la información del usuario que el sistema debe almacenar con fines adaptativos son: datos y características personales, perfil académico, perfil personal, características contextuales, historia y conocimiento de las tareas. En cuanto a la estructura organizacional del modelo de dominio y modelo pedagógico es considerado como lo plantea Arias Sánchez en su trabajo.

\section{ADAPTACIÓN}

Al proponer un sistema adaptativo, se debe establecer qué se va a adaptar, inicialmente se ha hablado de tipos de usuario (estudiante y turista) y estilos de aprendizaje.

Se estableció que la adaptación por tipo de usuario se implementará clasificando el orden de los contenido que vera cada uno, es decir, el tipo de usuario Estudiante obedecerá la estructura diseñada en el sistema, pero teniendo la posibilidad de escoger los temas o unidades que quiere aprender, con la condición de que debe de cumplir con conocimientos previos, ya que en éste se pretende un proceso de enseñanzaaprendizaje estructurado y acorde con lo visto en el aula de clases, además se requiere un mayor seguimiento del proceso de aprendizaje en este tipo de usuario. El tipo de usuario turista solo tendrá acceso al contenidos de las guías relacionadas con cada tema, es decir, que para éste se alterara el orden de la presentación de los contenidos sin que el turista tenga que ver la clasificación de cursos y unidades, solo vera las guías las cuales contienen el resumen de los temas, datos importantes e información orientada mayormente al turismo, dando mayor importancia al aprendizaje de los estudiantes y brindando al turista una forma de aprender y adquirir información de interés sin necesidad de que el proceso se vuelva tedioso.

El estilo de aprendizaje es uno de los aspectos que permite adaptar la forma como se presentan los contenidos del curso de acuerdo a las preferencias de cada estudiante, con el fin de facilitar la comprensión, también se implementa esta forma de adaptación mediante test de Felder y Silverman que determinan qué estilos de aprendizaje o preferencias predominan en una persona a la hora de aprender, por medio de éste proceso se establecerían los OA más acordes para el proceso de aprendizaje de cada estudiante. 
Finalmente, otro tipo de adaptación será la evaluación, se pretende que el sistema evalúe de forma adaptativa por medio de un TAI (Test Adaptativo Informatizado), de tal manera que a medida que el estudiante avanza en los niveles, se le harán preguntas y dependiendo de su respuesta, el sistema escogerá la siguiente pregunta hasta que éste alcance los logros para pasar al siguiente tema, la evaluación será interactiva, donde se tendrá un número limitado de equivocaciones aceptadas, si el estudiante llega a pasar éste número tendrá que repetir la lección o el tema evaluado.

\section{AGENTES}

Los actores identificados en la plataforma de aprendizaje adaptativa sobre turismo cultural e histórico en la ciudad de Santa Marta son: Estudiante, Turista, Planificador y Evaluador. Teniendo en cuenta que un agente puede ser un actor humano o una entidad de software, de los actores identificados se pueden establecer los siguientes agentes: Agente humano estudiante, Agente humano turista, Agente de software Profesor, Agente de software Estudiante, Agente de software turista, Agente de software Planificador, Agente de software Evaluador y Agente de software Interfaz.

Agente humano estudiante: Cumple el rol de aprendiz, es la persona que busca obtener conocimiento de la cultura e historia de Santa Marta para complementar lo aprendido en el aula de clases, cabe recordar que estos agentes son estudiantes pertenecientes a colegios públicos en la especialidad de turismo, los cuales adquieren conocimientos previos en el aula de clases.

Agente humano turista: Cumple el rol de aprendiz, es la persona que busca obtener conocimiento de la cultura e historia de Santa Marta con fines de ejercer turismo, se considera que no tienen un conocimiento previo de los temas por lo que se pretende que la plataforma haga el papel de guía, identificando intereses y ayudando al turista a conocer la ciudad de Santa Marta y su importancia histórica y cultural.

Agente de software profesor: Este agente se encargara de mantener organizada y estructurada la información correspondiente a los contenidos educativos del CVA (Modelo del Dominio), de tal manera que pueda ser utilizada por todos los agentes del sistema que la requieran.

Agente de software estudiante: Este agente se encargara de mantener organizada y estructurada la información correspondiente de los estudiantes (Modelo del estudiante), de tal manera que pueda ser utilizada por todos los agentes del sistema que la requieran.

Agente de software turista: Este agente se encargara de mantener organizada y estructurada la información correspondiente de los turistas (Modelo del turista), de tal manera que pueda ser utilizada por todos los agentes del sistema que la requieran.

Agente de software planificador: La principal función de este actor es adaptar un plan de estudio, para que el estudiante sea guiado por el sistema a través del proceso de enseñanza como si lo hiciera un profesor real o en el caso del turista como si fuera un guía turístico virtual, el cual tiene la capacidad de brindar una enseñanza individualizada. Otras actividades del planificador son: recuperar información académica y personal de los estudiantes y turistas y recuperar los resultados de las evaluaciones.

Agente de software evaluador: Este agente cumple la función de evaluar el desempeño del agente humano estudiante e intereses del agente humano turista.

\section{CONCLUSIÓN}

El trabajo bajo el cual está basado éste documento se encuentra en desarrollo. Fomentar un buen proceso de enseñanza-aprendizaje de la cultura 
e historia de la ciudad más antigua de América, ayudando a fortalecer el conocimiento de los estudiantes y dando a conocer los aspectos más importantes a los turistas de la ciudad de Santa Marta como fuente cultural e histórica de conocimiento es la motivación de éste trabajo. Se espera que bajo la orientación del modelo de dominio planteado en este artículo, el cual corresponde a la primera etapa de análisis del sistema tutorial inteligente orientado al proceso de enseñanza-aprendizaje de la cultura e historia de Santa Marta, Colombia, ayude a llegar a resultados que permitan obtener el diseño de un ambiente virtual que promueva una experiencia educativa y enriquecedora para sus usuarios. Al tiempo, se pretende motivar la realización de nuevas investigaciones y proyectos que tengan en cuenta en apoyo de las TICs en torno al impacto que tiene en las áreas de educación y el turismo.

\section{REFERENCIAS}

[1]. Francisco J. Arias, (2009) Modelo MultiAgente Para la Planificación Instruccional y Selección de Contenidos dn Cursos Virtuales Adaptativos. Universidad Nacional de Colombia - Sede Medellín.

[2]. Salcedo, P., Labraña, C., Farran, Y. (2002) Una Plataforma Inteligente de Educación a Distancia que Incorpora la Adaptabilidad de Estrategias de Enseñanza al Perfil, Estilos de Aprendizaje y Conocimiento de los Alumnos. infoUYclei, Congreso Uruguayo de Informática y Centro Latinoamericano de Estudios en Informática.
[3]. Pueyo, S. Empleo de una Plataforma On Line para la Integración de la Cultura en el Proceso de Aprendizaje de ELE. Universidad de Deusto. XIV Congreso Internacional de ASELE. Burgos 2003.

[4]. Cataldi, Z., Salgueiro, F., Lage, F., (2006) Sistemas tutoriales multiagentes con modelado del estudiante y del autor. Edutec, RevistaElectrónica de Tecnología Educativa, Num 20, Enero 06.

[5]. Cataldi, Zulma, et al. "Diseño de Sistemas Tutores Inteligentes con Tecnología de Agentes: Los Agentes Docentes en el Módulo Tutor." Revista Eletrônica de Sistemas de Informação ISSN 1677-3071 doi: 10.5329/RESI 6.1 (2007).

[6]. Francisco J. Arias, JovaniJiménez Builes, Demetrio A. Ovalle. (2008)Construcción de Cursos Virtuales Adaptativos conEnfasis en Aprendizaje Personalizado Activo. Grupo de Investigación y Desarrollo en Inteligencia Artificial. Universidad Nacional de Colombia - Sede Medellín.

[7]. Jimenez, J. (2002) Modelo de Integración de Ambientes Individuales y Colaborativos de Aprendizaje: Nuevo Paradigma Educativo. 